\section{The H3K27me3 demethylase JMJD3 contributes to the activation of the INK4A-ARF locus in response to oncogene- and stress-induced senescence}

\author{
Karl Agger, Paul A.C. Cloos, Lise Rudkjær, \\ Kristine Williams, Gitte Andersen, \\ Jesper Christensen, and Kristian Helin ${ }^{1}$
}

Biotech Research and Innovation Centre (BRIC) and Centre for Epigenetics, University of Copenhagen, DK-2200 Copenhagen, Denmark

The tumor suppressor proteins $\mathrm{p}^{\mathrm{INK} 4 \mathrm{~A}}$ and $\mathrm{p} 14^{\mathrm{ARF}}$, encoded by the INK4A-ARF locus, are key regulators of cellular senescence. The locus is epigenetically silenced by the repressive $\mathrm{H} 3 \mathrm{~K} 27 \mathrm{me} 3$ mark in normally growing cells, but becomes activated in response to oncogenic stress. Here, we show that expression of the histone $\mathrm{H3}$ Lys 27 (H3K27) demethylase JMJD3 is induced upon activation of the RAS-RAF signaling pathway. JMJD3 is recruited to the INK4A-ARF locus and contributes to the transcriptional activation of $\mathrm{p}^{\mathrm{INK} 4 \mathrm{~A}}$ in human diploid fibroblasts. Additionally, inhibition of Jmjd3 expression in mouse embryonic fibroblasts results in suppression of $\mathrm{p} 16^{\text {Ink4a }}$ and $\mathrm{p} 19^{\text {Arf }}$ expression and in their immortalization.

Supplemental material is available at http://www.genesdev.org.

Received October 20, 2008; revised version accepted March 27, 2009.

Senescence induced by oncogenes and other stress signals has been identified as a fail-safe program that counters malignant transformation (Serrano and Blasco 2007; Prieur and Peeper 2008). The tumor suppressor proteins p16 ${ }^{\mathrm{INK} 4 \mathrm{~A}}$ and $\mathrm{p} 14^{\mathrm{ARF}}$ are key regulators of senescence and the expression of these proteins leads to a G1 arrest of the cell cycle (Gil and Peters 2006). p16 ${ }^{\mathrm{INK} 4 \mathrm{~A}}$ causes this arrest by working as a CDK4/CDK6 inhibitor acting upstream of the pRB-E2F pathway, whereas p14 ${ }^{\mathrm{ARF}}$ (p19 ${ }^{\text {Arf }}$ in mice) controls the level of p53 by inhibiting the p53-specific ubiquitin ligase MDM2 (Gil and Peters 2006). In actively growing human diploid fibroblasts, the INK4A-ARF locus is silenced by histone H3 Lys 27 trimethylation (H3K27me3) imposed by the Polycomb Group (PcG) proteins. When such cells are exposed to cellular stress, the H3K27me 3 mark on the locus is decreased, resulting in expression of both $\mathrm{p} 16^{\mathrm{INK} 4 \mathrm{~A}}$ and $\mathrm{p} 14 /$ p19 ${ }^{\mathrm{ARF}}$ (Jacobs et al. 1999; Bracken et al. 2007; Kotake et al. 2007). In contrast, aberrant expression of PcG pro-

[Keywords: Cancer; senescence; INK4A; ARF; histone methylation; histone demethylation]

${ }^{1}$ Corresponding author.

E-MAIL kristian.helin@bric.ku.dk; FAX 45-3532-5669.

Article is online at http://www.genesdev.org/cgi/doi/10.1101/gad.510809. teins leads to increased H3K27me3 levels and silencing of the INK4A-ARF locus. The PcG proteins are frequently overexpressed in human tumors, and their contribution to proliferation is ascribed primarily to their role in regulating the expression of the INK4A-ARF locus (Jacobs et al. 1999; Bracken et al. 2007; Dietrich et al. 2007).

Recently, we and others identified two histone lysine demethylases-JMJD3 and UTX - that specifically catalyze the demethylation of di- and trimethylated H3K27 (H3K27me2/me3) (Agger et al. 2007; De Santa et al. 2007; Jepsen et al. 2007; Lan et al. 2007; Lee et al. 2007). We wanted to understand whether one or both of these enzymes could be involved in the active removal of H3K27me3 from the INK4A-ARF locus during induction of stress-induced senescence and oncogene-induced senescence (OIS).

\section{Results and Discussion}

IMJD3 expression is induced by activation of the RAS-RAF pathway

To investigate a potential role of JMJD3 and UTX in OIS, we generated TIG3-hTERT human diploid fibroblasts expressing a conditional form of the constitutively activated BRAF oncogene. This version of BRAF is fused to the ligand-binding domain of the estrogen receptor (ER), and the activity of this protein is rapidly induced by 4-hydroxytamoxifen (OHT) (Woods et al. 1997). These cells, TIG3 BRAF-ER, rapidly undergo OIS upon BRAF activation, concomitant with an increased expression of p16 ${ }^{\mathrm{INK} 4 \mathrm{~A}}$ (Fig. 1A,E). To understand whether JMJD3 and/ or UTX would be differentially expressed when cells enter OIS, we measured the expression levels of JMJD3 and UTX in response to BRAF activation by real-time quantitative PCR (qPCR) and Western blotting. As shown in Figure 1, B and E, the activation of BRAF leads to a significant increase in JMJD3 expression levels, whereas no change was observed in UTX expression.

If JMJD3 is required for the initial removal of the H3K27me3 mark before p16 ${ }^{\text {INK4A }}$ expression, one would expect that the increase in JMJD3 expression would occur prior to the increase in $\mathrm{p} 16^{\mathrm{INK} 4 \mathrm{~A}}$ expression. As shown in Figure 1, B and E, increased JMJD3 expression is already observed 2-4 $\mathrm{h}$ after activation of BRAF, whereas p16 ${ }^{\text {INK4A }}$ expression is not significantly increased before 24-48 h. In agreement with this, an increase in JMJD3 protein levels is already apparent $6 \mathrm{~h}$ after OHT treatment, whereas the increase in $\mathrm{p} 16^{\mathrm{INK} 4 \mathrm{~A}}$ protein is first noticeable after $24 \mathrm{~h}$ (Fig. 1E). Similar effects were obtained when we expressed BRAF-ER in BJ cells immortalized by hTERT (Fig. 1D).

Consistent with previous reports (e.g., see Bracken et al. 2007), levels of EZH2 and SUZ12, two subunits of the H3K27 methyltransferase complex PRC2, also decrease following the activation of BRAF (Fig. 1C,E). The decrease in EZH2 levels occurred later than the increase in JMJD3 expression, suggesting that JMJD3 may be affecting the transcription of the INK4A-ARF locus prior to the decrease of the PRC2 complex associated with the locus. From these data we conclude that JMJD3 expression is induced rapidly in response to BRAF activation and that this induction occurs before induction of INK4A 

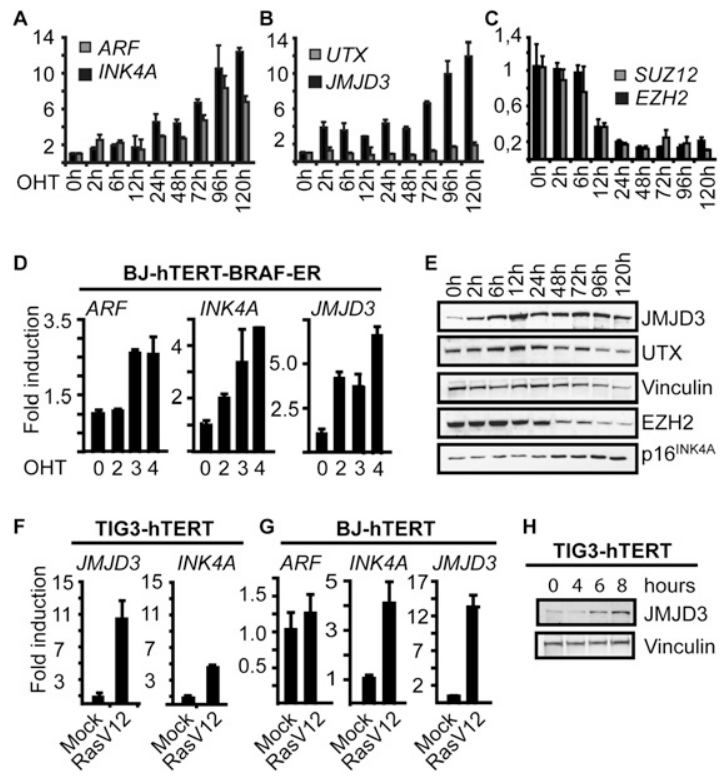

Figure 1. JMJD3 expression is induced by oncogenic BRAF and HRAS $^{\text {V12 }} \cdot(A-C)$ Expression levels of p16 $6^{\mathrm{INK} 4 \mathrm{~A}}$ and p14 ${ }^{\mathrm{ARF}}(A)$, JMJD3 and UTX $(B)$, and EZH2 and SUZ12 $(C)$ in TIG3 BRAF-ER at different times following OHT treatment determined by RT-qPCR. Data presented in $A-C$ are derived from the same experiment, but are representative of at least three independent experiments. $(D)$ Expression levels of INK4A, ARF, and IMJD3 in BJ BRAF-ER at different times $(0,2,3,4$ d) following OHT treatment, determined by RTqPCR. (E) Protein levels of JMJD3, p16 ${ }^{\mathrm{INK} 4 \mathrm{~A}}$, and EZH2 determined by Western blotting following the activation of BRAF. $(F, G)$ Expression levels of INK4A,ARF, and JMJD3 in TIG3-hTERT and BJhTERT cells transduced with HRAS ${ }^{\mathrm{V} 12}$-encoding retrovirus. After selection, expression levels were determined by RT-qPCR. $(H)$ Protein levels of JMJD3 in TIG3-hTERT cells at different time points after treatment with $50 \mathrm{~J} / \mathrm{m}^{2} \mathrm{UV}$ irradiation.

transcription. This indicates that JMJD3 could be involved in the initial demethylation of the INK4A-ARF locus in response to oncogenic stress.

To understand if other oncogenes, known to induce senescence, would induce the expression of JMJD3 prior to the activation of the INK4A-ARF locus, we monitored the effect of oncogenic HRAS ${ }^{\mathrm{V} 12}$. As demonstrated in Figure 1F, ectopic expression of HRAS ${ }^{\mathrm{V} 12}$ led to the activation of JMJD3 and p16 ${ }^{\text {INK4A }}$. A similar effect was also observed on $I N K 4 A$, but not on $A R F$, expression in BJ-hTERT cells infected with a HRAS ${ }^{\mathrm{V} 12}$-expressing virus (Fig. 1G). These results suggest that hyperactivation of the RAS-RAF pathway leads to the activation of the $I N K 4 A$ gene in human cells through the transcriptional induction of JMJD3, whereas the activation of $A R F$ is only observed by BRAF.

We also investigated if other types of stress associated with senescence can induce the expression of JMJD3, and as shown in Figure 1H, UV-irradiation of TIG3 human diploid fibroblasts led to a significant increase in JMJD3 protein levels. Taken together with the results reported above, this suggests that JMJD3 expression is increased in response to several types of stress.

JMJD3 contributes to the induction of $p 16^{I N K 4 A}$ expression in response to $B R A F$

Having established that JMJD3 is induced in response to oncogenic stress, we were interested in understanding the molecular mechanisms leading to JMJD3 activation. Previously, the transcription factor NF-кB has been implicated in the transcriptional activation of JMJD3 during inflammatory responses (De Santa et al. 2007). We speculated whether NF- $\mathrm{B}$ also could be involved in the induction of JMJD3 after oncogene stimulation. To investigate this, we tested whether the IkB $\alpha$-SR "superrepressor," a strong inhibitor of NF- $\mathrm{BB}$ signaling (Jobin et al. 1998), affected JMJD3 induction in response to BRAF. IkB $\alpha$-SR did not change the transcriptional activation of JMJD3 in response to BRAF (data not shown), indicating that NF- $\mathrm{KB}$ is not involved in the transcriptional activation of JMJD3 regulation in response to oncogene stimulation.

To gain mechanistic insight into how JMJD3 is induced in response to oncogenic stress, we cloned the human $J M J D 3$ promoter and established a reporter assay in U2OS cells expressing BRAF-ER. The JMJD3 promoter features two transcriptional start sites (TSSs) (Fig. 2B); one of which is used in embryonic stem (ES) cells (ESC-TSS), and another that is used in macrophages (MF-TSS) (De Santa et al. 2007). In response to BRAF activation, a promoter construct, P1, containing 3422 base pairs (bp) immediately upstream of the MF-TSS, is induced to a significantly higher level than the $J M J D 3$ reporter construct P8, containing 2928 bp upstream of the ESC-TSS
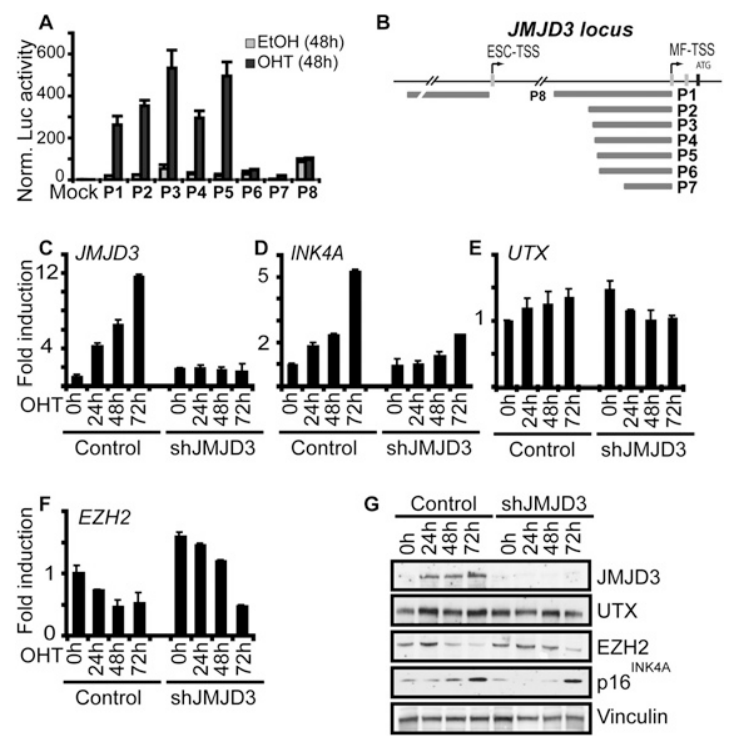

Figure 2. Induction of $\mathrm{p} 16^{\mathrm{INK} 4 \mathrm{~A}}$ expression in response to BRAF is regulated by JMJD3. (A) Reporter assays using lysates from BRAF-ER U2OS cells transfected with various human JMJD3 promoter/luciferase reporter gene constructs with or without OHT stimulation. OHT or mock was added $36 \mathrm{~h}$ after transfection, and cells were harvested after $48 \mathrm{~h}$ and assayed. $(B)$ Schematic representation of the human JMJD3 locus. Gray boxes indicate the size and position of the different promoter constructs (P1, P2, P3, etc.) used. Arrows indicate the ESC-TSS, and MF-TSS. (C-F) TIG3 BRAF-ER cells were transduced with shRNA plasmids targeting JMJD3 [shJMJD3(3302)] or control shRNA. Following selection, expression levels of $J M J D 3$, INK4A, UTX, and EZH2 mRNA were monitored by RT-qPCR after OHT treatment. $(G)$ The protein levels of JMJD3, p16 ${ }^{\text {INK4A, UTX, }}$ and EZH2 determined by Western blotting following OHT treatment. Data presented in $C-F$ are derived from the same experiment and are representative of at least three independent experiments. Error bars on qPCR experiments and reporter assays indicate standard deviations. 
start site (Fig. 2A,B). Reporter constructs P2, P3, P4, and P5, containing 2439, 2314, 2224, and 2173 bp immediately upstream of the MF-TSS, respectively, were also induced. The induction was lost in constructs P6 and P7 (spanning 2102 and 1428 bp upstream of the MF-TSS, respectively) indicating that the motifs crucial for BRAF induction of the JMJD3 promoter are found between 2102 and $2173 \mathrm{bp}$ upstream of the MF-TSS (Fig. 2B). This region of the promoter contains consensus sites for the following transcription factors: RAP1, Sp1, Krox-20, ETF, C/EBP $\alpha$, and AP-1. Notably, AP-1 is activated by the extracellular signal-regulated kinase (ERK), which regulates cell proliferation and is a component of the RAS-RAF-MEKERK kinase pathway. Hence, the AP-1 transcription factors are good candidates for acting as transactivators of $J M J D 3$ transcription in response to RAS-RAF-MEKERK signaling.

Since the increase in JMJD3 expression precedes the induction of $\mathrm{p} 16^{\mathrm{INK} 4 \mathrm{~A}}$ during BRAF-induced senescence, we wanted to understand if the increase in $\mathrm{p} 16^{\mathrm{INK} 4 \mathrm{~A}}$ expression was dependent on JMJD3. For this purpose, we generated two different lentiviral shRNA constructs encoding shRNAs that targeted JMJD3 expression (Supplemental Fig. S1A). We infected TIG3 BRAF-ER cells with these constructs as well as with a control shRNA construct selected for stable genomic integration, and measured the mRNA and protein levels of p16 ${ }^{\mathrm{INK} 4 \mathrm{~A}}$ and JMJD3 before and after BRAF induction. As shown in Figure 2, C and G, and Supplemental Figure S1A, both shRNAs to JMJD3 inhibited the expression of JMJD3. Importantly, this inhibition strongly reduced the induction of $\mathrm{p} 16^{\mathrm{INK} 4 \mathrm{~A}}$ expression following activation of BRAF (Fig. 2D,G; Supplemental Fig. S1B,C). Interestingly, JMJD3 depletion also appeared to increase EZH2 levels, perhaps reflecting an increased proliferative activity in JMJD3 knockdown cells. No change in UTX expression levels was observed (Fig. 2E-G). Thus, from these data we conclude that JMJD3 contributes significantly to the transcriptional activation of the INK4A-ARF locus. This is most likely a consequence of a lack of H3K27me3 demethylation at the locus resulting in transcriptional repression. However, consistent with the increased levels of $\mathrm{p} 16^{\mathrm{INK} 4 \mathrm{~A}}$ expression after sustained activation of B-RAF, even in the presence of an shRNA to JMJD3, down-regulation of JMJD3 was not sufficient to prevent OIS (data not shown).

Ectopic expression of JMJD3 results in the activation of the INK4A-ARF locus and the inhibition of cell proliferation

Next, having established that induction of $\mathrm{p} 16^{\mathrm{INK} 4 \mathrm{~A}}$ is dependent on JMJD3, we speculated whether ectopic expression of JMJD3 alone would be sufficient to induce transcription from the $I N K 4 A-A R F$ locus. Since we were not able to efficiently express full-length JMJD3 by retroviral transduction, we expressed a catalytically active fragment of JMJD3 (JMJD3s) in mouse embryo fibroblasts (MEFs). We transduced a panel of different primary MEFs, which were either wild type or carried mutations in genes involved in stress-induced senescence: $T p 53^{-/-}, A r f^{-1-}$, or Ink4a-Arf ${ }^{-1-}$, with viruses expressing JMJD3s or a catalytically inactive fragment of JMJD3s, JMJD3sMT. After transduction and $3 \mathrm{~d}$ of puromycin selection the expression levels of Ink $4 a$ and Arf were measured by RT-qPCR. As shown in Figure 3A,
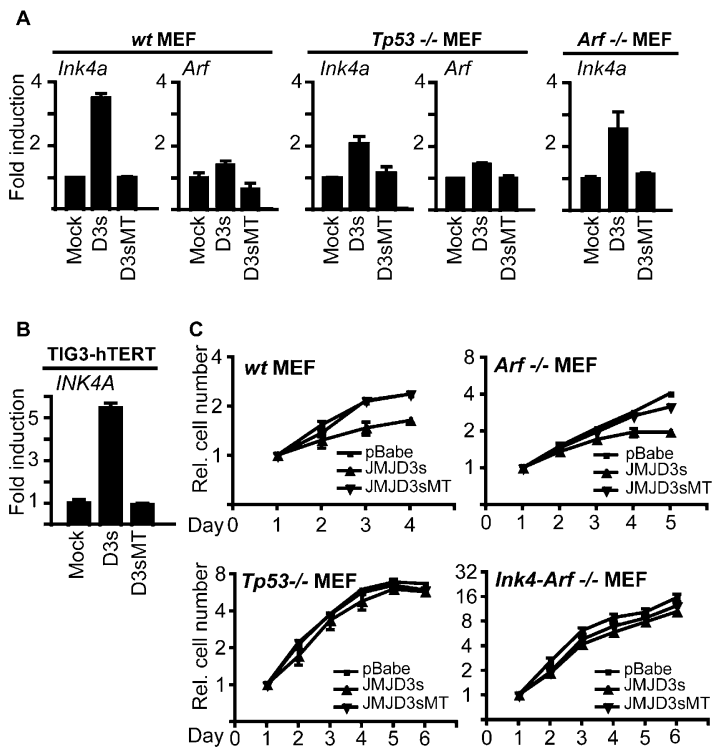

Figure 3. Overexpression of JMJD3 results in increased expression of INK4A in MEFs and TIG3 cells. (A) Wild-type, Tp53 ${ }^{-1-}$, Arf $^{-1-}$, and Ink4a-Arf ${ }^{-/}$cells were infected with pBabe constructs expressing a HA-tagged version of the catalytically active fragment of JMJD3 (JMJD3s), as well as the catalytically inactive version (JMJD3sMT, or D3sMT in figure). Mock refers to cells infected with empty pBabe vector. The expression levels of Ink4a and Arf were measured by RT-qPCR. Data are plotted as relative to mock-infected cells. (B) The expression level of INK4A was measured by RT-qPCR in TIG3-hTERT cells infected with mock, JMJD3s, and JMJD3sMT. Data are plotted as relative to mock-infected cells. (C) Growth curves using MEFs with the indicated genotypes infected with mock, JMJD3s, and JMJD3sMT. Y-axes are $\log 2$ scale.

ectopic expression of JMJD3s led to increased levels of Ink4a, but not Arf, in wild-type, Tp53 ${ }^{-/-}$, and $A r f^{-/-}$ MEFs. Since this effect was strictly dependent on the catalytic activity of Jmjd3 (Fig. 3A), these results suggest that Jmjd 3 can activate Ink $4 a$ expression by demethylating H3K27me3 associated with the locus. Similarly, we found that the activation of INK4A in human diploid fibroblasts was dependent on the catalytic activity of JMJD3 (Fig. 3B).

To investigate the consequence of increased JMJD3 activity, we measured the proliferation of different infected pools of MEFs (Fig. 3C). Expression of JMJD3s, but not of JMJD3sMT, strongly inhibited the proliferation of wildtype and $A r f^{-/-}$cells, again underscoring the importance of the demethylase activity of JMJD3 for its biological activity. Ectopic expression of JMJD3s, although expressed at much higher levels than in wild-type MEFs (Supplemental Fig. S2C), did not affect the proliferation

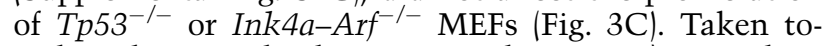
gether, these results demonstrate that JMJD3\ can induce the expression of $\mathrm{p} 16^{\text {Ink4a }}$ leading, to a strong inhibition of cell proliferation.

\section{Down-regulation of Imjd3 leads to immortalization of primary $M E F S$}

MEFs undergo stress-induced senescence after five to six passages, when grown in atmospheric $\mathrm{O}_{2}(20 \%)$ under a $3 \mathrm{~T} 3$ protocol. The stress response is a consequence of the high oxygen levels that inflict oxidative damage to the cells resulting in senescence (Parrinello et al. 2003). 
The induction of senescence is dependent on a functional Ink $4 a-A r f$ locus, although it is the p19 $9^{\text {Arf }}$ protein that mediates the response in mouse cells. Thus, inactivation of Arf is sufficient to overcome the stress-induced senescence process in MEFs (Kamijo et al. 1997). However, since the expression of the Ink4a and Arf genes is epigenetically coregulated (Bracken et al. 2007), we investigated the functional relevance of $\mathrm{Jmj} \mathrm{d} 3$ for the regulation of the entire Ink4a-Arf locus in MEFs.

To do this, we infected MEFs with a lentiviral construct that efficiently inhibited the expression of Jmjd3 (Supplemental Fig. S3A). The cells were subsequently subjected to a $3 \mathrm{~T} 3$ protocol and samples were harvested for protein preparations at the indicated times (Fig. 4A,B). As demonstrated in Figure 4 and Supplemental Figure S3B, depletion of Jmjd3 results in impaired expression of both $\mathrm{p} 16^{\text {Ink4a }}$ and $\mathrm{p} 19^{\text {Arf }}$ and a rescue of the senescence normally observed around passage 5-6.

As in human fibroblasts, MEFs also undergo OIS in response to activated oncogenes such as RasV12. To understand if Jmjd3 in mice has similar functions in OIS we transduced primary MEFs with lentiviruses expressing shJmjd3 and RasV12 as indicated in Figure 4, $\mathrm{C}$ and D. After selection, the expression levels of Ink $4 a$ and Arf were measured by RT-qPCR and immunoblotting. As demonstrated in Figure 4, C and D, depletion of Jmjd3 results in impaired expression of $\operatorname{Ink} 4 a$, as well as Arf, suggesting that mouse Jmjd3, as human JMJD3, contributes to the activation of the Ink $4 a-A r f$ locus during OIS.
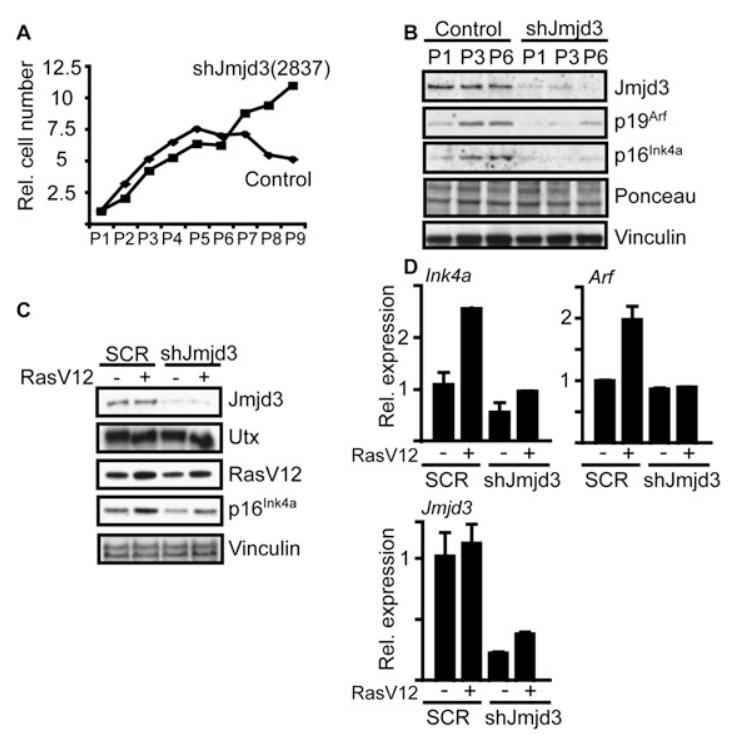

Figure 4. shRNA-mediated depletion of Jmjd3 inhibits stressinduced expression of $\mathrm{p} 16^{\text {Ink4a }}$ and $\mathrm{p} 19^{\text {Arf }}$ and leads to immortalization of primary MEFs. Primary MEFs were transduced with shRNA plasmids targeting Jmjd3 [shJmjd3(2837)] or control shRNA. (A) Jmjd3 depletion leads to immortalization of MEFs. The MEFs were passaged every $3 \mathrm{~d}$ and the relative cell number was plotted against the passage number. $(B)$ Western blotting of cell lysates prepared at the indicated passages using antibodies specific for the indicated proteins. (C) MEFs were coinfected with a lentiviral RasV12 expression construct and shJmjd3(2837) or control shRNA. (D) Cells were grown for 1-2 wk and expression levels of $\mathrm{p} 16^{\text {Ink4a }}$ p19 ${ }^{\text {Arf }}$, Jmjd3, and RasV12 were measured by immunoblotting and by RT-qPCR.

\section{JMJD3 associates with the INK4A-ARF locus}

Previous results have suggested that JMJD3 acts as a transcriptional coactivator by removing H3K27me3 at target genes (Agger et al. 2007; De Santa et al. 2007; Jepsen et al. 2007; Lan et al. 2007; Lee et al. 2007). Consequently, JMJD3 may contribute to the regulation of p $16^{\mathrm{INK} 4 \mathrm{~A}}$ and p14/p19 ${ }^{\mathrm{Arf}}$ expression by demethylating the INK4A-ARF locus. Previously, we mapped the binding of the PcG proteins and $\mathrm{H} 3 \mathrm{~K} 27$ trimethylation throughout the INK4A-ARF locus in human diploid fibroblasts, primary MEFs, and in cell populations enriched for hematopoietic stem cells (Bracken et al. 2007). The results from these studies showed that $\mathrm{H} 3 \mathrm{~K} 27 \mathrm{me} 3$ is associated primarily with the INK4A, and not the ARF, locus in the explanted fibroblasts. To understand if JMJD3 is associated with the INK4A-ARF locus in TIG3 BRAFER cells, we performed chromatin immunoprecipitation (ChIP) experiments followed by real-time qPCR (qChIP) using an antibody specific for JMJD3. As shown in Figure $5, A$ and $C$, the activation of BRAF leads to an increased binding of JMJD3 to regions just upstream of the TSS for $I N K 4 A$. Moreover, the increased binding correlates with a decrease in $\mathrm{H} 3 \mathrm{~K} 27 \mathrm{me} 3$ levels bound at the $I N K 4 A-A R F$ locus (Fig. 5B) and the decrease of the PcG protein CBX8 (Fig. 5C), a component of the Polycomb-Repressive Complex 1 that is required for the repression of the INK4A$A R F$ locus (Dietrich et al. 2007). These results show that JMJD3 directly binds to the INK4A-ARF locus and suggests that it contributes to the transcriptional induction of the locus by demethylating H3K27me3. If JMJD3 is responsible for the initial removal of $\mathrm{H} 3 \mathrm{~K} 27 \mathrm{me} 3$ on the INK4A-ARF locus in response to activation of the RASRAF pathway, the H3K27me3 levels would be affected by JMJD3 depletion. We performed ChIP experiments using TIG3-BRAF-ER cells and MEFs depleted for JMJD3 and expressing RasV12. The amount $\mathrm{H} 3 \mathrm{~K} 27 \mathrm{me} 3$ relative to total $\mathrm{H} 3$ at the INK4A locus was measured. As shown in Figure 5, D and E, depletion of JMJD3 impairs the $\mathrm{H} 3 \mathrm{~K} 27 \mathrm{me} 3$ reduction observed in response to oncogene activation.

\section{JMJD3 expression is significantly decreased in several types of primary tumors}

To obtain insight into whether deregulation of JMJD3 might have a role in cancer, we searched the Oncomine database (http://www.oncomine.org) for differential JMJD3 expression in normal versus tumor tissue. Notably, the expression of JMJD3 was significantly reduced relative to normal tissues in various cancers, including lung and liver carcinomas, as well as various hematopoietic malignancies (Fig. 5F; Supplemental Fig. S4). The most dramatic decrease of JMJD3 transcription was noted in a subset of lymphomas and leukemias. Thus, JMJD3 expression levels were reduced more than two standard deviations below the mean when compared with normal $\mathrm{B}(\mathrm{NB})$ cells in $64 \%$ (44 out of 69 ) of diffuse large B-cell lymphoma (DLBL) samples, in 62\% (19 out of 31) of Burkitt lymphomas (BL), in 50\% (eight out of 16) of hairy cell leukemias (HCL), and in all (four out of four) multiple myelomas (MM). In contrast, follicular lymphomas (FL), mantle cell lymphomas (MCL), and B-cell chronic lymphocytic leukemias (B-CLL) featured JMJD3 levels above or in the range of NB cells (Fig. 5F). These results suggest that decreased expression of JMJD3 might contribute to 
A
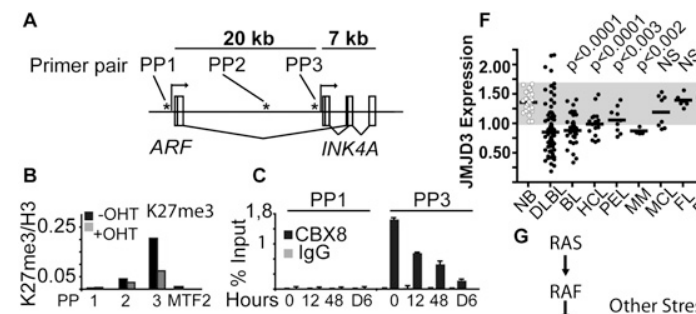

2.007.

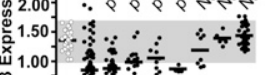

3

0.50 38. G RAS $\downarrow$
RAF

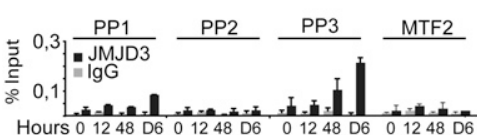
Stimuli MEK Stimul ERK

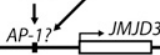

D TIG3-RAFER E MEF
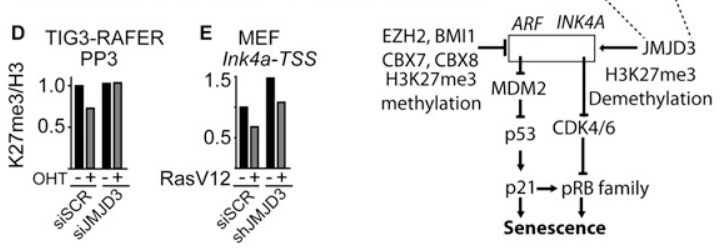

Figure 5. JMID3 associates with the INK4A-ARF locus. $(A)$ Schematic presentation of the INK4A-ARF locus with an indication of the primer pairs $(\mathrm{PP})$ used in $B-D .(B, C)$ ChIP assays using lysates from TIG3 BRAF-ER cells treated with or without OHT for the indicated time. In $C$, antibodies specific for JMJD3 or CBX8 were used; H3K27me3 was used in $B$. The degree of enrichment was measured by qPCR and results are presented as percentage of bound/ input; the H3K27me3 signal was normalized to the levels of histone $\mathrm{H} 3$ bound. The error bars represent the standard deviation of the mean. qPCR primers amplifying last exon of the MTF2 gene were used as negative control. (D) Changes in K27me3 levels at the INK4A-TSS in TIG3 BRAF-ER cells treated with siJMJD3 or control in response to OHT treatment for $48 \mathrm{~h}$. $(E)$ Changes in $\mathrm{H} 3 \mathrm{~K} 27 \mathrm{me} 3$ levels at the Ink4a-TSS in response to RasV12 expression with and without Jmjd3 knockdown in MEFs. $(F)$ Array data from Basso et al. (2005) reanalyzed to show expression levels of JMJD3 in NB cells and different types of lymphoma and leukemia including DLBL, BL, HCL, primary effusion lymphoma (PEL), FL, MM, MCL, and B-CLL (BLL in the figure). The shaded area indicates the mean \pm two standard deviations of controls (NB). Bars indicate the means; $P$-values of Mann-Whitney U-test are provided. $(G)$ Model for the regulation of the INK $4 A-A R F$ locus by JMJD3. See the text for details.

the development of some human cancers, most likely by reducing $\mathrm{H} 3 \mathrm{~K} 27$ demethylation of the INK4A-ARF tumor suppressor locus.

The INK4A-ARF locus is frequently lost in human cancers (Sherr 2001). The locus encodes two distinct tumor suppressor proteins: $\mathrm{p} 16^{\mathrm{INK} 4 \mathrm{~A}}$ and $\mathrm{p} 14^{\mathrm{ARF}}$ (Quelle et al. 1997; Sherr 2001). p16 $16^{\mathrm{INK} 4 \mathrm{~A}}$ and $\mathrm{p} 14^{\mathrm{ARF}} / \mathrm{p} 19^{\mathrm{ARF}}$ fulfill important roles in regulating the cell cycle and in the integration of stress pathways and cellular fates (Quelle et al. 1997; Sherr 2001). Thus, the p16 $6^{\mathrm{INK} 4 \mathrm{~A}}$ protein inhibits cell cycle progression through repression of the CDK4/6 cyclin complexes and by preventing the phosphorylation of $\mathrm{pRB}$, whereas the $\mathrm{p} 14^{\mathrm{ARF}}$ and p19 $9^{\mathrm{ARF}}$ proteins play important roles in regulation of the p53 pathway (Serrano et al. 1996; Kamijo et al. 1997).

In the present study, we showed that JMJD3 contributes to the normal activation of the INK4A-ARF tumor suppressor locus in response to stressful stimuli, advancing JMJD3 as a potential tumor suppressor gene. In agreement with this, we found that ectopic expression of JMJD3 leads to increased levels of $\mathrm{p} 16^{\mathrm{INK} 4 \mathrm{~A}}$ and senescence (Fig. 3; data not shown).

A key question is whether JMJD3 is mutated, deleted, or otherwise silenced/inactivated in cancers. As men- tioned above, data mining indicates that JMJD3 is decreased significantly in subsets of cancers (Fig. 5F). Moreover, as noted previously (Cloos et al. 2008), the $J M J D 3$ gene is located on chromosome 17 in close vicinity to the TP53 (p53) tumor suppressor gene. Allele loss at chromosome $17 \mathrm{p}$, where both TP53 and JMJD3 are found, constitutes a common genetic lesion observed in a variety of human cancers (Nigro et al. 1989). Interestingly, it has been proposed that this genomic area may include another tumor suppressor gene in addition to TP53, since allelic losses at this genomic position are rather poorly correlated with mutations in TP53. Thus, JMJD3 seems to be a strong candidate for this "additional" tumor suppressor gene. Indeed, loss of both TP53 and JMJD3 may be expected to inactivate both the pRB and p53 tumor suppressor pathways.

Another question that remains to be answered is what mechanisms lead to the transcriptional induction and recruitment of JMJD3 to the INK4A-ARF locus in response to oncogenic stress. The RAS/RAF/MEK/ERK pathway controls transcriptional programs, which can lead to different cellular outcomes such as apoptosis, transformation, cell proliferation, or senescence. Activated ERK, the downstream kinase in this pathway, can enter the nucleus and phosphorylate transcription factors such as c-JUN and c-FOS. These downstream transcription factors could regulate JMJD3. Indeed, the minimal promoter area necessary for JMJD3 induction in response to BRAF features a binding site for AP-1, a target of the ERK kinases.

The transcription factor AP-1 is formed as a dimer between members of the JUN family and members of the FOS or ATF families, and has been implicated in the control of transcription from the INK4A-ARF locus. Thus, c-JUN and FRA-1 proteins have been identified as major effectors of RAS-mediated transformation (Mechta et al. 1997). Similarly, AP-1 has been shown to play important roles in RAS-induced cellular transformation (Johnson et al. 1996). Finally, AP-1 heterodimers have been shown to cooperate with RAS to activate the ARF promoter (Ameyar-Zazoua et al. 2005). We therefore suggest a model (Fig. 5G) in which the ERK kinase acts to induce JMJD3 through a transcription factor, probably AP-1. In turn, JMJD3 demethylates H3K27 at the INK4A$A R F$ locus to induce expression of $\mathrm{p} 16^{\mathrm{INK} 4 \mathrm{~A}}$ and $\mathrm{p} 14^{\mathrm{ARF}} /$ p19 $9^{\mathrm{ARF}}$, thus fulfilling an important tumor suppressor function.

\section{Materials and methods}

\section{Data mining and statistical analysis}

Microarray data from Oncomine Web site (http://www.oncomine.org) were compared between groups using the Mann-Whitney U-test (two-tailed). For all tests, $P \leq 0.05$ was considered significant.

\section{ShRNA constructs}

The following shRNA constructs against mouse Jmjd3 were in the LKO.1-puro vector from Sigma: shJmjd3(5876), TRCN000 0095264; and shJmjd3(2837), TRCN0000095265. ShRNA constructs against human JMJD3 were cloned in the flap-Hygro vector targeting the following sequences: shJMJD3(3302), GGCG ACAGAAGGAGCATCA; and shJMJD3(4705), GATGATCTCT ATGCATCCA. 


\section{Generation of antibodies to JMJD3}

The JMJD3 antibody was generated in rabbits, using affinitypurified GST-JMJD3 (amino acids 798-1095). The antisera were affinity-purified on GST-JMJD3.

\section{Other antibodies}

Anti-H3 (ab1791, Abcam); anti-H3K27me3 (\#9733, Cell Signaling); anti-Vinculin (V9131, Sigma); anti-RasV12 (Sc-520, Santa Cruz Biotechnologies); anti-CBX8 (Bracken et al. 2007); antiEZH2 (BD43) (Bracken et al. 2007); anti-HA (MMS-101P, Covance); anti-INK4A (DCS50); anti-Ink4a (Santa Cruz Biotechnologies).

\section{Generation of BRAF-ER TIG3 cells and MEFs}

Generation of BRAF-ER TIG3 cells and MEFs is described in the Supplemental Material.

\section{ChIP and real-time $q P C R$}

ChIP and real-time qPCR were done essentially as described in Bracken et al. (2007). Primers used are described in the Supplemental Material.

Cloning of the JMJD3 promoter, reporter assays, and analysis of potential transcription factor-binding sites

Cloning of the JMJD3 promoter, reporter assays, and analysis of potential transcription factor-binding sites isdescribed in the Supplemental Material.

\section{Acknowledgments}

We thank members of the Helin laboratory for technical advice and fruitful discussions. We thank Jesus Gil and Gordon Peters for communicating results prior to submission. This work was supported by grants from the Novo Nordisk Foundation, the Association for International Cancer Research, the Danish Cancer Society, the Danish Medical Research Council, the Danish Natural Science Research Council, the Benzon Foundation, the Lundbeck foundation, Harboefonden, and the Danish National Research Foundation.

\section{References}

Agger K, Cloos PA, Christensen J, Pasini D, Rose S, Rappsilber J, Issaeva I, Canaani E, Salcini AE, Helin K. 2007. UTX and JMJD3 are histone H3K27 demethylases involved in HOX gene regulation and development. Nature 449: 731-734.

Ameyar-Zazoua M, Wisniewska MB, Bakiri L, Wagner EF, Yaniv M, Weitzman JB. 2005. AP-1 dimers regulate transcription of the p14/ p19ARF tumor suppressor gene. Oncogene 24: 2298-2306.

Basso K, Margolin AA, Stolovitzky G, Klein U, Dalla-Favera R, Califano A. 2005. Reverse engineering of regulatory networks in human B cells. Nat Genet 37: 382-390.

Bracken AP, Kleine-Kohlbrecher D, Dietrich N, Pasini D, Gargiulo G, Beekman C, Theilgaard-Monch K, Minucci S, Porse BT, Marine JC, et al. 2007. The Polycomb group proteins bind throughout the INK4A-ARF locus and are disassociated in senescent cells. Genes \& Dev 21: 525-530.

Cloos PA, Christensen J, Agger K, Helin K. 2008. Erasing the methyl mark: Histone demethylases at the center of cellular differentiation and disease. Genes \& Dev 22: 1115-1140.

De Santa F, Totaro MG, Prosperini E, Notarbartolo S, Testa G, Natoli G. 2007. The histone H3 lysine-27 demethylase Jmjd3 links inflammation to inhibition of polycomb-mediated gene silencing. Cell 130: 1083-1094.

Dietrich N, Bracken AP, Trinh E, Schjerling CK, Koseki H, Rappsilber J, Helin K, Hansen KH. 2007. Bypass of senescence by the polycomb group protein CBX8 through direct binding to the INK4A-ARF locus. EMBO J 26: 1637-1648.

Gil J, Peters G. 2006. Regulation of the INK4b-ARF-INK4a tumour suppressor locus: All for one or one for all. Nat Rev Mol Cell Biol 7: $667-677$.

Jacobs JJ, Kieboom K, Marino S, DePinho RA, van Lohuizen M. 1999. The oncogene and Polycomb-group gene bmi-1 regulates cell proliferation and senescence through the ink4a locus. Nature 397: 164-168.

Jepsen K, Solum D, Zhou T, McEvilly RJ, Kim HJ, Glass CK, Hermanson O, Rosenfeld MG. 2007. SMRT-mediated repression of an H3K27 demethylase in progression from neural stem cell to neuron. Nature 450: 415-419.

Jobin C, Panja A, Hellerbrand C, Iimuro Y, Didonato J, Brenner DA, Sartor RB. 1998. Inhibition of proinflammatory molecule production by adenovirus-mediated expression of a nuclear factor $\kappa \mathrm{B}$ super-repressor in human intestinal epithelial cells. J Immunol 160: 410-418.

Johnson R, Spiegelman B, Hanahan D, Wisdom R. 1996. Cellular transformation and malignancy induced by ras require c-jun. Mol Cell Biol 16: 4504-4511.

Kamijo T, Zindy F, Roussel MF, Quelle DE, Downing JR, Ashmun RA, Grosveld G, Sherr CJ. 1997. Tumor suppression at the mouse INK4a locus mediated by the alternative reading frame product p19ARF. Cell 91: 649-659.

Kotake Y, Cao R, Viatour P, Sage J, Zhang Y, Xiong Y. 2007. pRB family proteins are required for $\mathrm{H} 3 \mathrm{~K} 27$ trimethylation and Polycomb repression complexes binding to and silencing p16INK $4 \alpha$ tumor suppressor gene. Genes \& Dev 21: 49-54.

Lan F, Bayliss PE, Rinn JL, Whetstine JR, Wang JK, Chen S, Iwase S, Alpatov R, Issaeva I, Canaani E, et al. 2007. A histone H3 lysine 27 demethylase regulates animal posterior development. Nature 449: 689-694.

Lee MG, Villa R, Trojer P, Norman J, Yan KP, Reinberg D, Di Croce L, Shiekhattar R. 2007. Demethylation of H3K27 regulates polycomb recruitment and H2A ubiquitination. Science 318: 447-450.

Mechta F, Lallemand D, Pfarr CM, Yaniv M. 1997. Transformation by ras modifies AP1 composition and activity. Oncogene 14: 837-847.

Nigro JM, Baker SJ, Preisinger AC, Jessup JM, Hostetter R, Cleary K, Bigner SH, Davidson N, Baylin S, Devilee P, et al. 1989. Mutations in the p53 gene occur in diverse human tumour types. Nature 342: 705708.

Parrinello S, Samper E, Krtolica A, Goldstein J, Melov S, Campisi J. 2003. Oxygen sensitivity severely limits the replicative lifespan of murine fibroblasts. Nat Cell Biol 5: 741-747.

Prieur A, Peeper DS. 2008. Cellular senescence in vivo: A barrier to tumorigenesis. Curr Opin Cell Biol 20: 150-155.

Quelle DE, Cheng M, Ashmun RA, Sherr CJ. 1997. Cancer-associated mutations at the INK4a locus cancel cell cycle arrest by p16INK4a but not by the alternative reading frame protein p19ARF. Proc Natl Acad Sci 94: 669-673.

Serrano M, Blasco MA. 2007. Cancer and ageing: Convergent and divergent mechanisms. Nat Rev Mol Cell Biol 8: 715-722.

Serrano M, Lee H, Chin L, Cordon-Cardo C, Beach D, DePinho RA. 1996. Role of the INK4a locus in tumor suppression and cell mortality. Cell 85: 27-37.

Sherr CJ. 2001. The INK4a/ARF network in tumour suppression. Nat ReV Mol Cell Biol 2: 731-737.

Woods D, Parry D, Cherwinski H, Bosch E, Lees E, McMahon M. 1997. Raf-induced proliferation or cell cycle arrest is determined by the level of Raf activity with arrest mediated by p21Cip1. Mol Cell Biol 17: 5598-5611. 


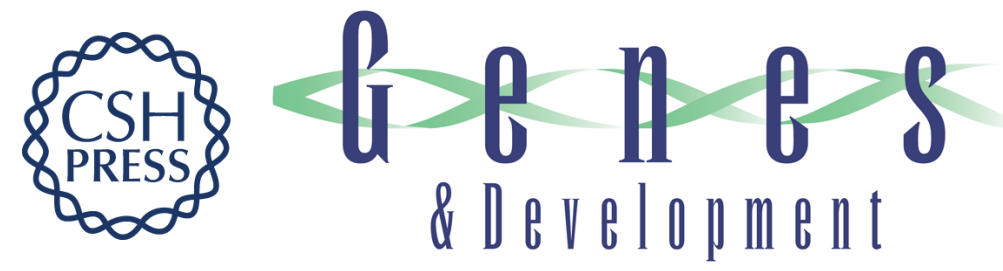

\section{The H3K27me3 demethylase JMJD3 contributes to the activation of the INK4A-ARF locus in response to oncogene- and stress-induced senescence}

Karl Agger, Paul A.C. Cloos, Lise Rudkjær, et al.

Genes Dev. 2009, 23:

Access the most recent version at doi:10.1101/gad.510809

Supplemental http://genesdev.cshlp.org/content/suppl/2009/05/07/23.10.1171.DC1

Material

References This article cites 25 articles, 8 of which can be accessed free at:

http://genesdev.cshlp.org/content/23/10/1171.full.html\#ref-list-1

License

Email Alerting Receive free email alerts when new articles cite this article - sign up in the box at the top

Service right corner of the article or click here.

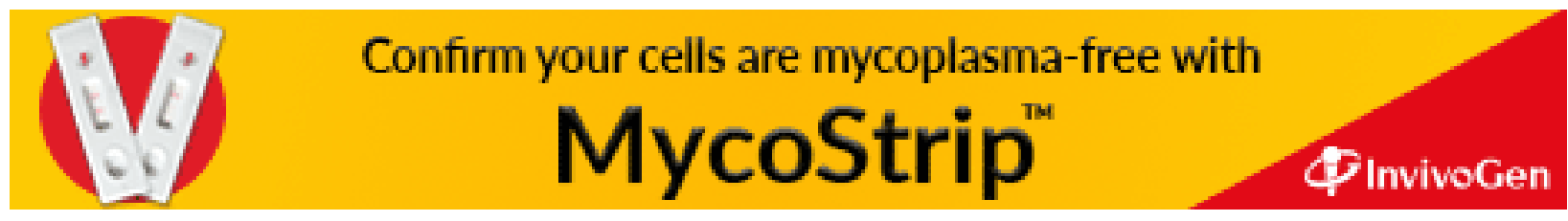

